\title{
Positioning and identification of markers for 3D tracking
}

\author{
Olivier Magneau ${ }^{\mathrm{a}}$, Patrick Bourdot and Rachid Gherbi \\ Action transversale "VENISE", LIMSI-CNRS, Université Paris XI, BP 133, 91403 Orsay Cedex, France
}

Received 30 June 2003, Accepted 20 October 2003

\begin{abstract}
This paper addresses the positioning and identification of markers for 3D tracking within Virtual Reality (VR) applications. Many VR applications need to know the position and the orientation of the body of users in order to make possible intuitive user interactions as stereoscopy calculation, navigation control or gesture dialog. This work is based on infrared capture device that does not need to be physically dependent with tracked objects. During an acquisition, some markers could be occluded by objects or not visible by some cameras. This paper proposes a new solution that finds the position of the occluded markers or at least limits the consequences of oclusion. The aim is to minimise the effects of oclusion by an optimal distribution of the markers on the objects. It also uses a fast and robust markers identification algorithm. The global approach is based on the association of markers distribution with their identification, in order to optimise the number of visible markers.
\end{abstract}

Key words: 3D tracking / marker positioning / marker identification / virtual reality

Résumé - Positionnement et identification de marqueurs pour le suivi 3D. Cet article porte sur le positionnement et l'identification de marqueurs pour le suivi 3D dans des applications de Réalité Virtuelle. Ces applications nécessitent souvent la connaissance de la position et de l'orientation du corps des utilisateurs afin de permettre des interactions utilisateur intuitives comme le calcul adaptatif de la stéréoscopie, la commande de la navigation ou le dialogue gestuel. Ce travail est basé sur un dispositif de capture infrarouge qui n'a pas besoin d'être lié physiquement aux objets à suivre. Lors d'une acquisition, certains marqueurs peuvent être occultés par des objets ou non visibles par les caméras. Cet article propose une nouvelle approche qui détermine la position des marqueurs occultés ou au pire limite les conséquences de ces occultations. Le principe est de minimiser les effets de l'occultation par une distribution optimale des marqueurs sur les objets. L'approche repose également sur un algorithme rapide et robuste d'identification de marqueurs. Le fonctionnement global est basé sur l'association de la distribution de marqueurs et de leur identification, afin d'optimiser le nombre de marqueurs visibles.

Mots clés : Suivi 3D / positionnement de marqueurs / identification de marqueurs / réalité virtuelle

\section{Introduction}

The Virtual Reality systems have a grown need to know the position and the orientation of users' body in order to make possible a realistic interaction between users and Virtual Environment. For example, the head parameters are necessary to compute an exact stereoscopy images [1] and free-hand navigation [2]. Another example concerns arms and hands parameters used in sign language [3], grasping and pointing [4]. These parameters are measured using various 3D tracking devices such as electromagnetism, ultrasonic or visible optics. These devices are based on technologies [5] that are often intrusive

\footnotetext{
${ }^{a}$ Corresponding author: magneau@limsi.fr
}

and they constrain the user's movements as shown in Figure 1a.

Since recently, 3D tracking devices based on infrared are less intrusive than previous devices. Indeed, infrared devices do not need to be physically connected with the tracked object as shown in Figure 1b. In the same way, visible optics have same characteristic as infrared ones. However, strong constraints on environment lighting intensity and weak acquisition rates considerably restrict the use of such device.

Infrared devices are well adapted for the tracking of users in immersive environment (Fig. 2). Currently, the only real-time device (Vicon) is very expensive and is only useful for applications which require to track biological movements. In the case of interactive applications, real-time is essential. For instance, when a user moves 


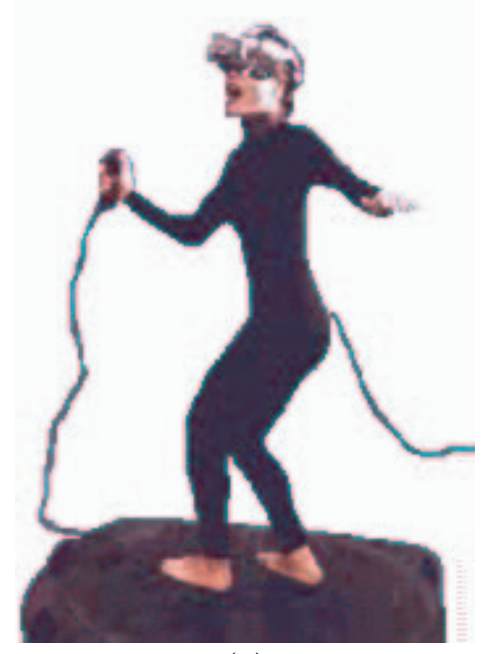

(a)

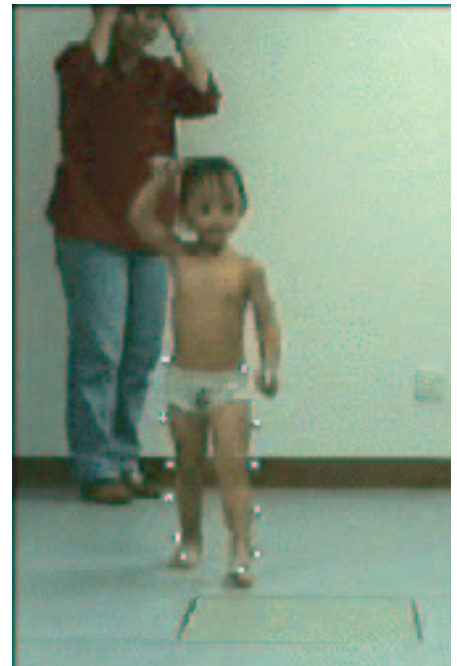

(b)

Fig. 1. Two examples of 3D tracking devices: (a) $\operatorname{Spacepad}^{\mathrm{TM}}$ (b) Vicon ${ }^{\mathrm{TM}}$.

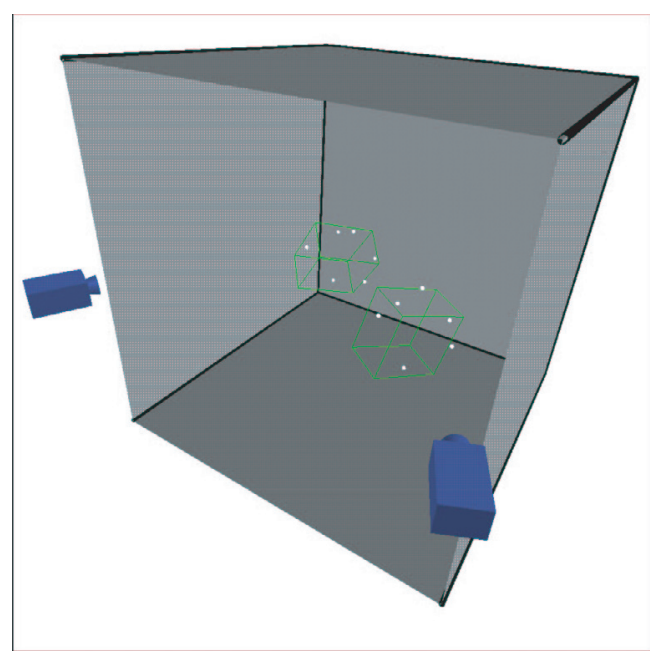

Fig. 2. Example of Virtual Reality system where 3D objects are tracked by 2 infrared cameras.

his head, stereoscopic images must be computed instantaneously.

Some systems based on infrared locate and calculate the $3 \mathrm{D}$ position of markers placed on the tracked objects. But the fact that these devices are monochromatic implies that it is not possible to discriminate the markers by their "colour". The only way to identify them is to exploit information on their relative positions to the object. This is a first problem that can be resolved by proposing a new positioning model of markers (see Sect. 5).

During an acquisition, some markers can be occulted or are not visible by a sufficient number of cameras [6] The classical solution for this problem is to increase the number of cameras. This is acceptable in the major cases, but is not possible in Virtual Environment. In our case, the positioning of cameras is limited. Consequently, this limits the number of cameras and their vision field.
This increases the occlusion probability of the markers. This second problem is addressed in Section 4 by implementing a new algorithm based on identification of markers.

The both positioning model and identification algorithm share a common functional module (see Sect. 3). This module aims to compute the probabilistic matrix that indicates the similarity between two lists of markers. In the positioning model, lists are identical and correspond to the markers on the object. In the identification, first list contains the markers on the object and second one the markers in space.

First, we introduce the general approach of the complete system in Section 2 and the common functional module "Discrimination algorithm" is presented. After, we describe in details both the identification algorithm and the positioning process. Finally, some results are shown.

\section{General approach}

Our approach is based on the association of both positioning of markers on objects and the identification of them. However, the positioning is a process that must be performed before. Moreover, in the processing, the segmentation algorithm uses the 3D position of all markers whereas others processing algorithms are applied for each object as shown in Figure 3.

In a first time, a pre-processing step is applied on each object in order to optimise the number of markers visible by the cameras and to produce data for the identification algorithm. Then, during the acquisition, the processing step is used. A segmentation algorithm is used to distinguish the various objects in the space. Besides, this algorithm creates the list of markers belonging to each object. After, we apply the various algorithms allowing us to compute the global parameters for each object. 


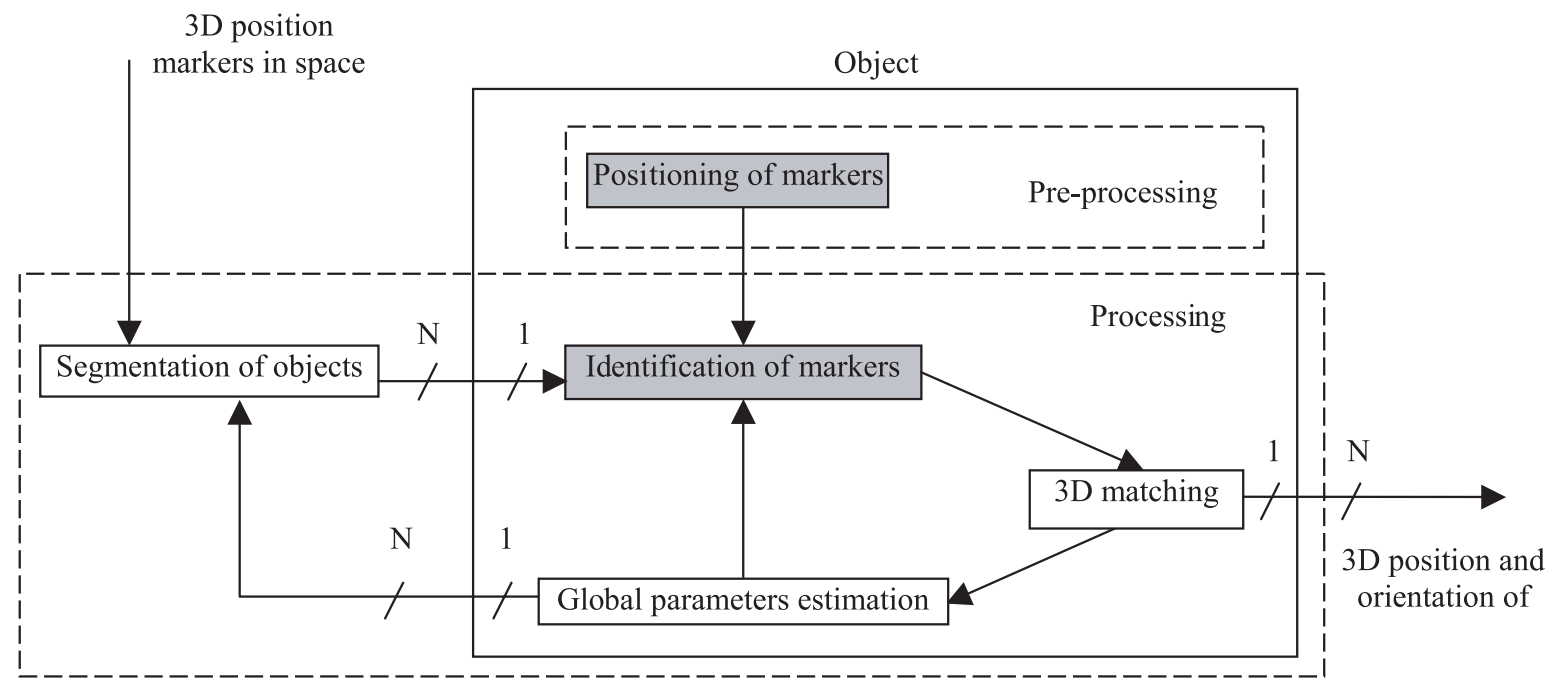

Fig. 3. Functional graph of operation of the tracking of $3 \mathrm{D}$ objects by infrared cameras.

The first one is the identification algorithm that associates the segmented markers with the positioning markers.

The second one is the 3D matching algorithm that computes the global parameters of the object. The finally one, the estimation algorithm is used to predict the global parameters of the object for the next acquisition time. This algorithm is useful for the segmentation to separate the various objects and to provide for the identification an initialisation state about the markers of this object. Figure 3 shows the various parts of this approach, as well as the links between these parts. In the following, main algorithms are described.

\section{Discrimination algorithm}

This algorithm allows the system to discriminate the various triangles of one object composed by triplets of its markers. This discrimination is represented by a coefficient that will be useful for both the positioning and identification algorithms. The discrimination algorithm computes the probability that two markers are similar. So, it computes the shape parameter and the size parameter for all possible triangles formed by all markers. Afterwards, it produces a correspondence table with these parameters. This table is used in both positioning and identification algorithms. This algorithm is developed in [7].

\section{Identification algorithm}

This algorithm takes as input data provided by both the segmentation and the global parameters estimation. The segmentation provides the list of markers that must be some members of the object. The estimation provides the estimated position of object's markers. A first part allows making a list of pre-identified markers (Sect. 4.1). Afterwards, the identification part uses this pre-identification to minimize the number of calculation
(Sect. 4.1). This algorithm uses the markers' position, given by the positioning process, to have the knowledge of the relative position of markers.

\subsection{Pre-identification algorithm}

This part estimates the final identification. To do that, it searches to link the real markers provided by the segmentation with the estimated markers (those corresponding to position calculated by estimation module). The positioning process provides the lower length $D$ between two markers of an object. This length is used to define a neighbourhood around each marker.

We aim here at minimising the number of calculation. So, instead of search what is the estimate marker the more near (or close) of a real marker, we search an estimate marker that is in the neighbourhood, which the size is define by $a D$, where $a$ is a value define by the user and strictly less than $1 / 2$.

The first advantage of this algorithm is its capability to stop searching other solutions, as soon as it finds one estimated marker in the neighbourhood of the real marker. The second advantage is due to the infrared device. In fact, the device provides a list of markers that are visible. If the same markers are visible for both an acquisition and the next one, then the position of markers in the list is not changed. If one marker or more are occluded, they are deleted from the list. If one marker or more are occluded to an acquisition and become visible to the next acquisition then they are placed to the end of the list. So if there is no occluded or dis-occluded marker from an acquisition to the next, then the pre-identification is found when the search is done in using the previous identification. In this case, this algorithm verifies that the previous identification and estimation modules are good.

The pre-identification algorithm is very quick in the ideal case. However, it is not necessary valid. This is why it'll have to use the identification process to validate or change the pre-identification. 

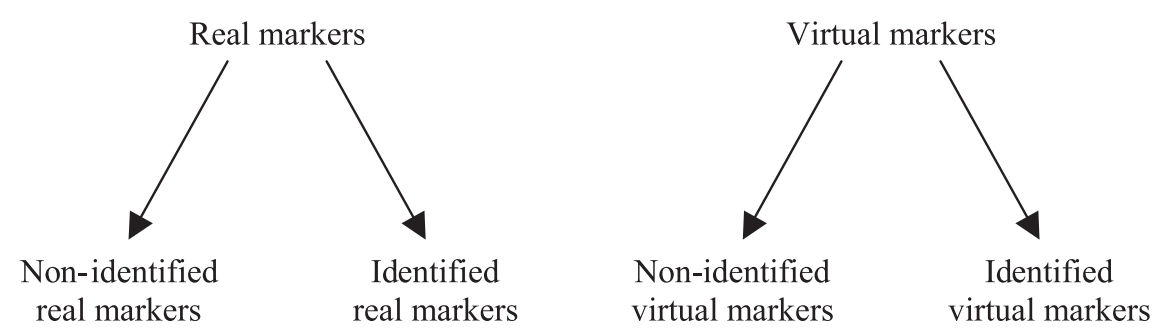

Fig. 4. Graph of various kinds of markers.

3 real markers 3 virtual markers

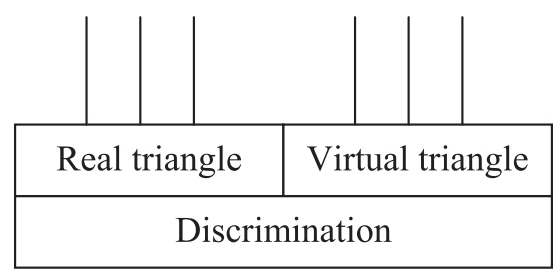

Fig. 5. Principle's graph of discrimination of two triangles.

\subsection{Identification process}

This process allows identifying the real markers with markers of object. It uses the discrimination algorithm to compare two triangles. This process has been chosen for that the triangles used can identified the markers. We define the notion of identification levels (see Sect. 4.2.1) that are used in the identification rules (see Sect. 4.2.2).

This process takes as input data the list of real markers and the list of virtual markers. The pre-identification algorithm allows associating some real markers with the corresponding virtual markers. So there are two kinds of markers by list: the identified markers and the nonidentified markers. Figure 4 shows the various kinds of markers.

The principle of the identification is to discriminate all combinations of three real markers (real triangles) with all combinations of three virtual markers (virtual triangles). Figure 5 shows the discrimination between real triangles and virtual triangles.

\subsubsection{Identification levels}

We define 4 identification levels from 0 to 3 . The level corresponding to the number of identified markers used in the real and virtual triangles to discriminate it. The identification of level 0 uses 3 non-identified real markers and 3 non-identified virtual markers as shown in Figure 6a. The identification of level 1 uses 2 non-identified real markers and 1 identified real marker for the real triangle and the corresponding identified virtual marker and 2 non-identified virtual markers for the virtual triangle as shown in Figure 6b. The identification of level 2 uses 1 non-identified real marker and 2 identified real markers for the real triangle and the corresponding identified virtual markers and 1 non-identified virtual marker for the virtual triangle as shown in Figure 6c. The identification of level 3 uses 3 identified real markers and the corresponding identified virtual markers as shown in Figure 6d.

After an identification level, it has to calculate the identification. To do that, we use the normalised correspondence table described in [5]. We create an identification list that allows linking each real marker with the associated virtual marker. This list is filled by searching the greater value of the normalised correspondence table. To finish, it calculates the number of values that are greater than a threshold value. We call it the identifying criterion. This number corresponding to the number of identified markers. The identifying criterion allows determining if the calculated identification is valid or not.

\subsubsection{Identification rule}

The identification rule determines what is the identification level to use in function of the previous state and the number of identified markers as shown in Figure 7 . In fact, if the number of identified markers is less than 2 , it is not possible to use the identification of level 3. Moreover, if the number of non-identified markers is less than 2 , it is not possible to use the identification of level 0 . So, the identification rule manages this kind of situation and determined if the identification is valid or if not, then it is necessary to continue in order to decide what identification level should be used.

The principle of the identification rule is to use the well-adapted identification level in function of identified markers number $N b I$. At the beginning, identification level corresponding to the number $N b I$ is used. If $N b I$ is greater than 3, it uses the identification level 3. Each identification level works in the same manner. If the new $\mathrm{NbI}$ value is lower than the level number, then the adapted identification level is used. If the new value is equal to the level number then it uses the lower one. In other cases, it leaves the process and validates the identified markers.

Finally, if the number of identified markers is lower than 3 then it is necessary to complete the identification in order to obtain at least 3 identified markers. To do that, it should complete the identified markers list with the identification that was considered as bad by the identification criterion.

The identification criterion is defined in order that identification level could decide how many markers are 


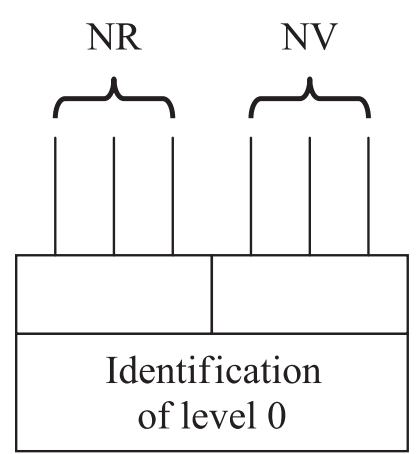

(a)

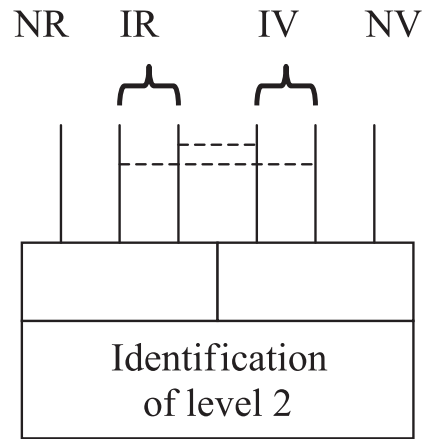

(c)

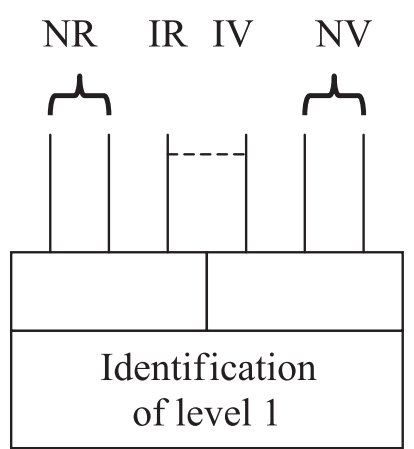

(b)

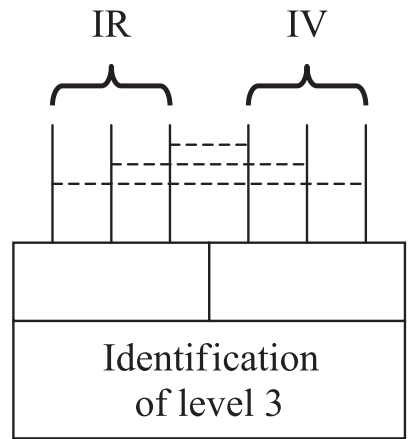

(d)

Fig. 6. Scheme representation the various identification levels.

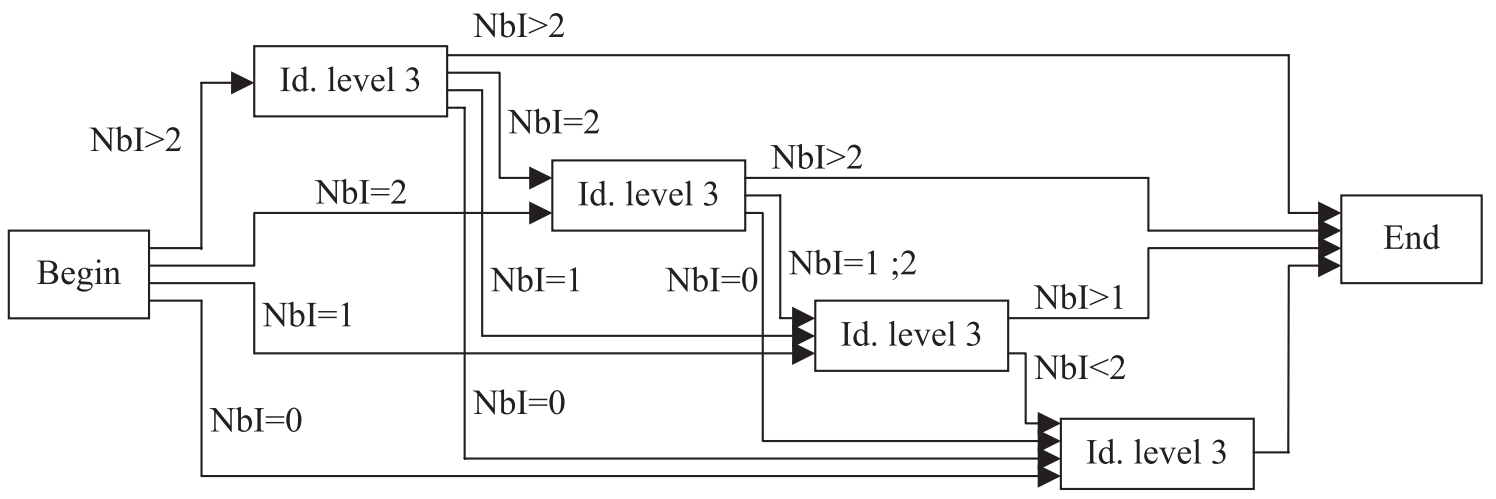

Fig. 7. Principle scheme of identification rule.

identified. By experimentation, the value that allows optimising the identification is $\sqrt{2}$.

\section{Positioning process}

The principle of the positioning is based on the computation of the optimal position of the objects markers. In this way, the triangles formed by the various triplets of markers are all different in shape and/or in size. The input of this algorithm is the 3D shape of the object and the number of markers and the output is the $3 \mathrm{D}$ position of markers and the value of the discrimination parameters $\alpha_{L}$ and $\alpha_{S}$.
This process is an iterative algorithm that executes the module of calculation of $3 \mathrm{D}$ position of markers followed by the module of calculation of the discrimination parameters while the value of the discrimination parameters become stable. The 3D position of markers is provided by displacement of markers (see Sect. 5.1) and the discrimination parameters value is processed by the corresponding module (see Sect. 5.2).

\subsection{Displacement marker process}

The goal of this process is to calculate a vector of displacement by marker. Two vectors compose these vectors: the vectors of shape (see Sect. 5.1.1) and size 


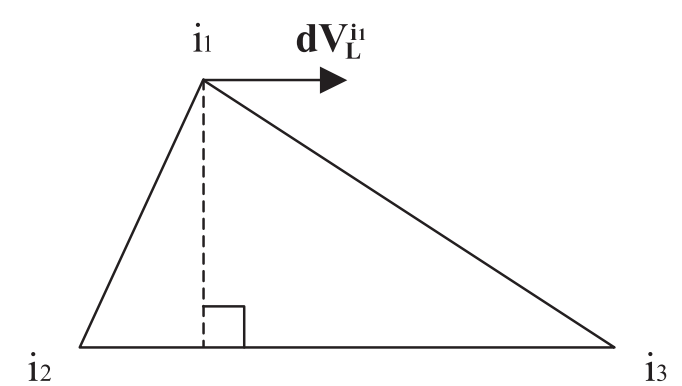

Fig. 8. Example of shape displacement vector.

(see Sect. 5.1.2) displacement. The resulting vector allows displacing the position of the markers. The resulting position is then projected to the object for that the markers stay on the object.

\subsubsection{Shape displacement}

This process is applied to all triangles $i$ formed by markers of object. We calculate the shape coefficient between the triangles $i$ and $j$ (this process is applied for all triangles $j \neq i$ ). For each marker of the triangle $i$, we calculate the vector of shape displacement $V_{L}^{i}$ so that the size of triangle do not changed. We achieved it by moving the markers parallel to the opposite side as shown by Figure 8 .

The norm of the vector $\boldsymbol{V}_{\boldsymbol{L}}^{\boldsymbol{i 1}}$ is provided by the shape coefficient previous calculated and it direction is done by the sign of the difference between' the lengths of triangles $i$ and $j$. In Figure 8, we can see that if the displacement is in the direction of the vector $\boldsymbol{V}_{\boldsymbol{L}}^{\boldsymbol{i} \mathbf{1}}$, the length $l_{23}$ between $i_{2}$ and $i_{3}$ is not changed, but $l_{12}$ is increased and $l_{13}$ is decreased.

In this context, in order to accentuate the value of shape parameter, the corresponding direction is chosen. This is applied to the other markers $i_{2}$ and $i_{3}$ of this triangle $i$.

\subsubsection{Surface displacement}

For all triangles formed by markers of object, we calculate the surface coefficient between the triangles $i$ and $j$. For each marker of the triangle $i$, we calculate the vector of surface displacement $\boldsymbol{V}_{\boldsymbol{S}}^{\boldsymbol{i}}$ so that the surface of triangle do not changed. We achieved it by moving the markers so that the shape is not changed as shown by Figure 9 .

The norm of the each vector is done by the surface coefficient of the triangle $i$ and the direction of each vector is the director vector of the straight line that goes through the corresponding marker and the centre of the circumscribed circle of the triangle. In Figure 9, we can see that if the displacement is in the direction of the vectors is out if the surface of the triangle $i$ is greater than the surface of triangle $j$. Else, the direction of the vectors is in the triangle. Like the shape displacement, we want to accentuate the value of surface parameter.

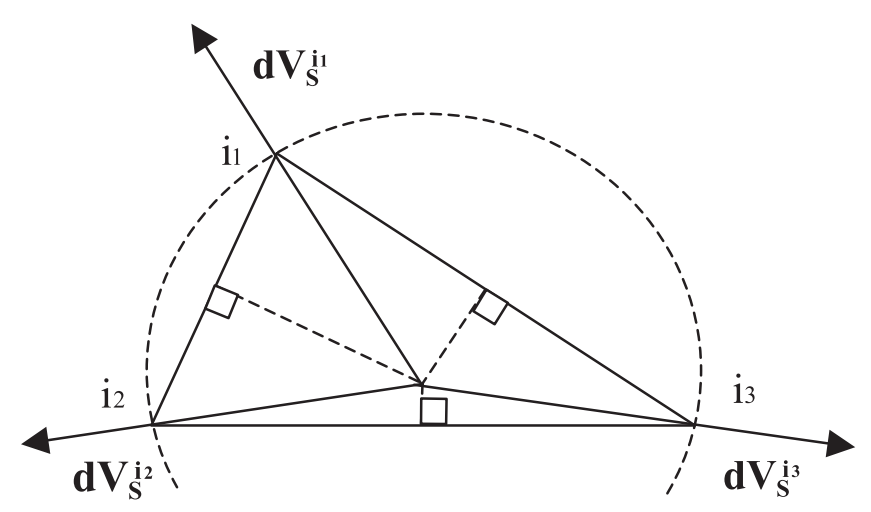

Fig. 9. Example of Surface displacement vectors.

\subsection{Calculation of discrimination parameters}

This part allows to calculate the optimal discrimination parameters $a_{L}$ and $a_{S}$. These parameters represent the value of the acceptable tolerance between the shape and the size of two triangles. So we can calculate these values by searching the minimum of a criterion that is defined by the ratio between the shape and the surface coefficients for the value of $a_{L}$ and the surface and the shape coefficients for the value of $a_{S}$.

Let us take the calculation of $a_{L}$ (the calculation of $a_{S}$ is the same). We search a criterion that allows to take account the minimum value of shape of all triangles when surfaces are equivalent and not take account the value of shape when surfaces are not equivalent. So the criterion used here is defined by the equation (1) and we search the minimum value of this criterion

$$
C_{\alpha L}^{i j}=\frac{P_{L}^{i j}}{P_{S}^{i j}}
$$

$P_{L}^{i j}$ and $P_{S}^{i j}$ are the shape and the surface coefficients defined in [5]. The minimum value of this criterion is equal to $C_{\alpha L}$

We suppose here that $C_{\alpha L}$ is equal to $P_{L}$ (the corresponding $P s$ is supposed equal to 1$)$. By definition, $P_{L}=e^{-\frac{C_{L}}{\alpha_{L}^{n}}}$, where $a_{L}^{n}$ is the value of $a_{L}$ at the time $n$.

At the time $n+1$, we pose that $a_{L}^{n+1}$ is equal to $\frac{C_{L}}{2}$. The solution is done by the equation (2)

$$
\alpha_{L}^{n+1}=\frac{-\alpha_{L}^{n} \cdot \ln \left(C_{\alpha L}\right)}{2}
$$

\section{Results}

In order to validate the identification, the experiment used in our work was defined by using a box on which have been positioned six markers (one marker by side). During the acquisition, we have observed many occlusions. Moreover, when the infrared device computes the $3 \mathrm{D}$ position of some markers, we observed some calculations errors. Tables 1 and 2 shows the number of identified markers in function of the number of real markers and the mean 
Table 1. Number of identified markers in function of number of real markers.

\begin{tabular}{ccccc} 
& \multicolumn{4}{c}{ Number of identified markers } \\
\hline $\begin{array}{c}\text { Number of } \\
\text { real markers }\end{array}$ & 3 & 4 & 5 & 6 \\
\hline 3 & 17 & 0 & 0 & 0 \\
\hline 4 & 23 & 46 & 0 & 0 \\
\hline 5 & 15 & 53 & 135 & 0 \\
\hline 6 & 0 & 20 & 35 & 156 \\
\hline
\end{tabular}

Table 2. Mean square error (MSE) and standard deviation (SD) in mm between estimated markers and those in space.

\begin{tabular}{ccccc}
\hline \multirow{2}{*}{$\begin{array}{c}\text { Number } \\
\text { of markers }\end{array}$} & \multicolumn{2}{c}{ All markers } & \multicolumn{2}{c}{ Identified markers } \\
\cline { 2 - 5 } & MSE & SD & MSE & SD \\
\hline 3 & 5.95 & 2.19 & 3.57 & 1.47 \\
4 & 6.53 & 3.85 & 2.77 & 0.70 \\
5 & 8.42 & 4.89 & 3.37 & 0.67 \\
6 & 5.17 & 2.45 & 3.48 & 0.35 \\
\hline
\end{tabular}

square error and standard deviation between estimated markers and those in space.

We can conclude that the number of identified markers is not always equal to the number of real markers, but the error of position of identified markers is lower than the error of all real markers. This implies that the identification is efficient and it is robust because the number of identified markers is more often equal to the number of real markers. When the number of identified markers is lower than the number of real markers, we can remark that the calculation of 3D position of markers in space is false. This is due to bad identification of markers in the infrared device, but this does not affect the identification of our identification algorithm.

\section{Conclusion and prospects}

This paper addressed the positioning and identification of markers for 3D tracking within Virtual Environment applications. We presented a new approach to track objects based on infrared cameras. During the corpora acquisition with Vicon ${ }^{\mathrm{TM}}$ device, several markers were occluded. This paper proposed a new solution that finds the position of the occluded markers or at least limits the consequences of occlusion. The identification algorithm provided good results. The results shown in previous section are achieved with a not optimal computing of the positioning of markers.

The identification is currently done on all triangles formed by the markers. However, we can notice that if two markers or more are occulted, then these markers are spatially closed. This encourages us to think that it is possible to decrease the number of triangles to be compared by eliminating the triangles formed by sufficiently distant markers. Besides, there are some differences between the $3 \mathrm{D}$ position of markers obtained by the positioning algorithm and the real $3 \mathrm{D}$ position of markers. It would be useful to compute the real $3 \mathrm{D}$ position of markers.

\section{References}

[1] C. Cruz-Neira, D.J. Gandin, T.A. DeFanti, Surroundscreen projection-based virtual reality: The design and implementation of the CAVE. In ACM SIGGRAPH 93 Proceedings, 1993, pp. 135-142

[2] P. Bourdot, D. Touraine, Polyvalent display frame work to control virtual navigations by $6 \mathrm{DOF}$ tracking, in IEEE Virtual Reality Conference 2002, IEEE VR 2002, Orlando, Florida (US), March 2002

[3] A. Braffort, R. Gherbi, S. Gibet, J. Richardson, D. Teil, Gesture-Based Communication in Human-Computer Interaction. International Gesture Workshop, GW'99, Gifsur-Yvette, France, March 1999, Lecture Notes in Artificial Intelligence No. 1739

[4] S. Augustine Su, Richard Furuta, A specification of 3D manipulation in virtual environments, in 1993 IEEE Annual Virtual Reality International Symposium, 1993, pp. 3837-393

[5] A. Mulder, Human movement tracking technology. Technical report 94-1, School of Kinesiology, Simon Fraser University, 1994

[6] R. Boulic, M.-C. Silaghi, D. Thalmann, Visualization of local movements for optimal marker positioning, in $A M D O$, 2000, pp. 133-144

[7] O. Magneau, P. Bourdot, R. Gherbi, 3D tracking based on infrared cameras, in International Conference on Computer Vision and Graphics, Zakopane, Poland, September 2002 